\title{
Non-invasive brain stimulation therapies
}

\author{
Terapias de neuroestimulação não invasivas
}

\author{
Paulo J. C. Suen ${ }^{1}$, Andre R. Brunoni ${ }^{1}$
}

Suen PJC, Brunoni AR. Non-invasive brain stimulation therapies / Terapias de neuroestimulação não invasivas. Rev Med (São Paulo). 2019 July-Aug;98(4):279-89.

\begin{abstract}
Noninvasive brain stimulation therapies are a promising field for the development of new protocols for the treatment of neuropsychiatric disorders. They are based on the stimulation of neural networks with the intent of modeling their synaptic activity to adequate levels. For this, it is necessary to precisely determine which networks are related to which brain functions, and the normal activation level of each of these networks, so that it is possible to direct the stimulation to the affected networks in order to induce the desired effects. These relationships are under intense investigation by the scientific community, and will contribute to the advancement of treatments by neurostimulation, with the emergence of increasingly accurate and effective protocols for different disorders. Currently, the most used techniques are Transcranial Direct Current Stimulation and Transcranial Magnetic Stimulation, with the most common applications being for treating Major Depressive Disorder. The advancement of research in this field may determine new target networks for stimulation in the treatment of other disorders, extending the application of these techniques and also our knowledge about brain functioning.
\end{abstract}

Keywords: Transcranial magnetic stimulation/standards; Implantable neuroestimulators; Neuropsychiatry; Nervous system diseases/therapy; Nervous system diseases/diagnosis.
RESUMO: As terapias biológicas não invasivas se apresentam como um campo promissor para o desenvolvimento de novos protocolos de tratamento de transtornos neuropsiquiátricos. Elas se baseiam na estimulação de redes neurais com intuito de modular sua atividade sináptica para níveis adequados. Para isso, é necessário a determinação precisa de quais redes estão relacionadas a quais funções cerebrais, e do nível de ativação normal de cada uma dessas redes, para que então seja possível direcionar a estimulação às redes afetadas a fim de induzir os efeitos desejados. Essas relações estão sob intensa investigação pela comunidade científica, e vão contribuir para o avanço dos tratamentos por neuroestimulação, com o surgimento de protocolos cada vez mais precisos e efetivos para diferentes transtornos. Atualmente, as técnicas mais utilizadas são a Estimulação Transcraniana por Corrente Contínua e a Estimulação Magnética Transcraniana, sendo a aplicação mais comum no tratamento do Transtorno Depressivo Maior. O avanço das pesquisas possivelmente determinará novas redes alvo para estimulação no tratamento de outros transtornos, estendendo a aplicação dessas técnicas e também do nosso conhecimento sobre o funcionamento cerebral.

Descritores: Estimulação magnética transcraniana/normas; Neuroestimuladores implantáveis; Neuropsiquiatria; Doenças do sistema nervoso/terapia; Doenças do sistema nervoso/diagnóstico.

1. Faculdade de Medicina FMUSP da Universidade de São Paulo. Serviço Interdisciplinar de Neuromodulação, Laboratório de Neurociências (LIM-27), Instituto de Psiquiatria - HC-FMUSP. ORCID: Brunoni AR - https://orcid.org/0000-0002-6310-3571. Email: paulo.suen@fm.usp.br; brunowsky@gmail.com.

Correspondence: André R. Brunoni. Instituto de Psiquiatria da FMUSP. Rua Dr. Ovídio Pires de Campos, 785. Cerqueira César - São Paulo, SP. CEP: 05403-903. Email: brunowsky@gmail.com. 


\section{INTRODUCTION}

$\mathrm{T}$ he use of electric current as a form of treatment for neurological diseases dates back to Classical Antiquity, from the earliest documentation of medical practice. De Compositionie Medicamentorum, by Scribonius Largus, Roman Emperor Claudius' physician, describes in detail a set of substances and recipes used by physicians of the time, and includes the use of electric rays for the treatment of headache and pain ${ }^{1,2}$. The etymology of the name of these electric rays in English, torpedo fish, is indicative of this origin: the word "torpedo" comes from the Latin "torpere" meaning numb.

The end of the eighteenth century was marked by the introduction of electrophysiology as a scientific discipline, beginning with Galvani's findings in 1791 of the electrical excitability of the nerves ${ }^{3,4}$. This notion was expanded to the cerebral cortex with the works of Fritsch and Hitzig $(1870)^{5}$, and other researchers of the time ${ }^{6-9}$, whose investigations produced conclusive evidence of the functional relationship of different brain regions through mapping with electrical stimuli ${ }^{10}$.

It was in the 1960s that scientists began investigating the modulation of neuronal activity by electrical current of a specific polarity. Bindman et al. ${ }^{11}$ showed that currents of $0.25 \mu \mathrm{A} / \mathrm{mm}^{2}$ applied to the pia mater influenced the spontaneous activity and evoked potentials of neurons for hours after only minutes of electrical stimulation in rat brains. Purpura and McMurtry ${ }^{12}$ observed the same phenomenon in preparations of cats with currents of $20 \mu \mathrm{A}$ $/ \mathrm{mm}^{2}$ applied to the cerebral cortex. These experiments indicate that currents far below those required to evoke an action potential can alter the excitability of neurons, and create the theoretical basis for Transcranial Electrical Stimulation (TES).

In 1985, Barker et al. ${ }^{13}$ introduced the Transcranial Magnetic Stimulation (TMS), after solving the technical challenges associated with the creation of a pulse of magnetic field of sufficient intensity to penetrate the skull that could generate significant induced electrical currents in the brain circuits. They showed that a magnetic pulse applied to the motor cortex was able to cause muscle contraction generating a motor evoked potential (MEP) measured by electromyography (EMG).

Currently, neurostimulation is divided into a broad field of research with different applications. Unlike invasive therapies such as deep brain stimulation (DBS) and vagus nerve stimulation (VNS), or convulsive therapies such as electroconvulsive therapy (ECT) and magnetoconvulsive therapy (MCT), non-invasive brain stimulation therapies do not require sedation or anesthesia, whilst being able to modulate cortical activity and excitability of neurons through the intact skull. Its two main stimulation techniques are repetitive TMS (rTMS) and TES, the most widespread variant of which is Transcranial Direct Current Stimulation
(tDCS), reintroduced in 2000 in its modern form[14].The aim of this chapter is to present, in a precise and succinct manner, the noninvasive stimulation therapies, rTMS and tDCS, and convulsive therapies, ECT and MCT. We will show the theoretical bases of each of them, explaining their mechanisms of functioning, as well as the emerging clinical applications for psychiatric disorders. Then, we will finish with a record of the main challenges faced and the future of these applications for psychiatry.

\section{MECHANISMS OF ACTION}

\section{Transcranial Magnetic Stimulation (TMS)}

TMS is based on the principle of induction of electric current in a circuit from a magnetic field as shown by Michael Faraday in $1832^{15}$. It uses a coil in which an electric current is generated, which in turn generates a pulse of magnetic field, called a field coil. The generated field has a direction perpendicular to the field coils, and reaches an intensity similar to that produced by magnetic resonance (1.5-3T) devices, but for a short period of time (milliseconds) ${ }^{16}$. An electric field is induced in the brain circuits affected by the magnetic field. The induced field voltage may itself excite neurons, but induced currents are likely to be the most important for the observed effects ${ }^{17}$. In a homogeneous medium, the electric current is induced in circuits parallel to the field coil, which in the brain translates into directional currents mainly tangential to the surface of the skull. The higher current circuits will be those with a similar circumference to the coil, with the induced current decreasing as it approaches its center ${ }^{17}$. The ions dissolved in the cytosol of the neurons are displaced with the appearance of electric field induced by the field coil, this displacement being larger according to the field gradient generated within the neuronal element. It is of our interest that this field gradient passes through the neuronal membrane in order to induce current flow through it and subsequent activation of the neuron.

The excitatory and inhibitory effects generated by rTMS are similar to the effects of long-term potentiation (LTP) and long-term depression (LTD). LTP and LTD are two mechanisms of synaptic plasticity caused by biological phenomena that lead either to enhancement (LTP) or weakening (LTD) of the synapse, causing an increase or decrease of its efficiency. Among the observed similarities, we highlight: 1) rTMS induces effects that last beyond the stimulation period; 2) the temporal pattern of stimulation is important for determining the physiological effect; 3) the effects of rTMS depend on the previous state of the stimulated neural network; 4) rTMS is involved in the expression of brain-derived neurotrophic factor (BNDF) and c-fos, LTP-associated molecules; 5) the effects of rTMS are decreased by blocking N-methyl D-aspartate (NMDA) receptors; and 6) the effects of rTMS are associated with BDNF polymorphisms ${ }^{18,19}$. 


\section{Transcranial Current Stimulation (tDCS)}

TDCS is technically one of the simplest ways to stimulate the brain. It consists of the passage of electric current of low intensity through electrodes placed on the scalp in order to provoke alterations in the cerebral activity modifying the excitability of the affected regions. The injected current alters the resting membrane potential, hyperpolarizing it or depolarizing it, according to the direction of the current in relation to the axonal orientation, without, however, provoking activation ${ }^{11,12}$. In this sense, the technique is purely neuromodulatory, different from TMS which, in addition to being neuromodulatory, is also neurostimulatory ${ }^{2}$. In general, anodic currents increase neuronal excitability, demonstrated by an increase in MEP amplitude to TMS excitation, while cathodic currents decrease it. Stimulation lasting for a few seconds is sufficient to induce such excitability changes, which however do not last beyond the stimulation period ${ }^{20}$. An effective modulation of the neuronal activity that lasts for at least one hour requires a stimulation of minutes ${ }^{21}$.

The long-term effects of tDCS are probably related to several different mechanisms. Pharmacologically, evidence indicates that these effects are related to the synaptic plasticity of glutamatergic neurons, since the blockade of NMDA receptors reduces the effects of $\mathrm{tDCS}^{22,23}$. TDCS has also been shown to reduce the local concentration of gamma aminobutyric acid (GABA), which may also impact glutamatergic plasticity given the close relationship between these two neurotransmitters [24]. TDCS can also influence the functional connectivity of the neural networks, impacting their oscillations and the synchronization between them ${ }^{25,26}$. There are also non-synaptic effects, since the presence of an electric field can cause the displacement of molecules involved in transmembrane ion transport, in the cell's membrane structure, cytoskeleton or substance transport mechanisms ${ }^{27,28}$.

The classic anode-excitatory, cathode-inhibitory rationale is valid for the motor cortex, but cannot be generalized to the whole brain. For example, it was observed in a study with healthy volunteers that the expected "AeCi" (anode-excitatory / cathode-inhibitory) effects occurred in only $1 / 3$ of the cases, with $40 \%$ of cases having excitatory effects with anode and cathode and, in $21 \%$ of cases, the effects were reversed (anode-inhibitory / cathode-excitatory ${ }^{29}$. In addition, a greater intensity of electrical current for longer periods may improve the effectiveness of the technique in some applications ${ }^{30}$, but this too cannot be considered a general rule. In fact, it was observed that by doubling the current intensity to $2 \mathrm{~mA}$ (vs $1 \mathrm{~mA}$ ) the cathode produced excitatory effects, rather than the expected inhibitory effects ${ }^{31}$, whereas the anodic stimulation at $1 \mathrm{~mA}$ for long periods led to inhibitory effects $^{32}$. In part, these effects can be explained by the fact that an increase in current intensity creates an electric field that penetrates more into the brain, and becomes capable of influencing the activity of deeper neural networks, which in turn impacts the biological and clinical effects ${ }^{33}$.

Another thing to be considered is that even if the injected currents induce depolarization or hyperpolarization coherent with the polarity of the electrodes, the observed physiological effects depend on the function of the affected network, which in turn can exert excitatory or inhibitory effects on the rest of the brain. Finally, the size of the electrodes and their arrangement on the scalp directly influence the diffusion of current and the geometry of the electric fields induced in the brain ${ }^{34,35}$. Thus, the variability of the clinical results observed with tDCS treatments may be directly related to anatomical differences between the individuals' brains, which alter the magnitude and direction of the electric field resulting from the stimulation ${ }^{36,37}$.

\section{Electroconvulsive therapy and magnetoconvulsive therapy (ECT and MCT)}

ECT and MCT induce convulsion within a controlled environment in patients under general anesthesia via depolarization of a large number of neurons. ECT is performed by placing two electrodes on the head that discharge an electric current of low duration and high intensity ${ }^{38}$, while the MCT applies magnetic pulses that depolarize the neurons of the affected region ${ }^{39}$. The higher focality of the electric fields induced by MCT is possibly related to a lower impairment of cognitive functions, such as spatial disorientation and memory loss, than the convulsions induced by ECT, while maintaining the therapeutic capacity of the technique ${ }^{40}$. Nevertheless, ECT has a history of more than 80 years of application in psychiatry, and is considered one of the most effective therapies for mood disorders, while MCT is still a treatment under investigation ${ }^{38}$.

The mechanisms by which convulsive therapy work are still poorly understood and most of the evidence comes from studies of ECT in depression41. The inflammation theory is promising, since we have observed a decrease in inflammatory cytokines in depressed patients after $\mathrm{ECT}^{42,43}$. Inflammation activates the kinurenin pathway, leading to oxidative stress and serotonin depletion ${ }^{44}$, thus a marked reduction in inflammation could decrease depressive symptoms ${ }^{45}$. Other lines show that ECT increases levels of $\mathrm{BDNF}^{46}$, hippocampal and amygdala volume $\mathrm{e}^{47,48}$ and functional connectivity of the hippocampus ${ }^{49}$.

\section{STIMULATION PARAMETERS}

\section{Transcranial Magnetic Stimulation (TMS)}

TMS can produce different effects and its applicability differs depending on the region of the brain being stimulated, whether this region is engaged in some activity or not, and the frequency, intensity and cycle of the generated magnetic pulses. In general, single-pulse and paired-pulse TMS are used to investigate brain functioning, 
Suen PJC, Brunoni AR. Non-invasive brain stimulation therapies.

while rTMS is used to induce changes in brain activity that extend beyond the stimulation period ${ }^{50}$.

The combination of single-pulse and paired-pulse TMS with EMG measurements makes it possible for us to investigate various processes of the motor cortex, such as excitability, plasticity, cortical connectivity, as well as interactions between excitatory and inhibitory cortical processes ${ }^{51}$. In addition, it is also a valuable tool for understanding the pathological processes related to neurological and psychiatric disorders such as Parkinson's disease, Alzheimer's disease, schizophrenia, obsessive compulsive disorder, major depressive disorder and bipolar disorder $^{52}$.

RTMS is used as a therapeutic tool for inducing long-term excitatory or inhibitory effects in brain circuits, with applicability to treat neurological and psychiatric disorders characterized by dysfunction in specific neural networks. This modulation of cortical activity is mainly dependent on stimulation frequency, with low frequencies $(\leq 1 \mathrm{~Hz})$ associated with an inhibitory effect to motor cortex excitability ${ }^{53}$, and high frequencies $(5-20 \mathrm{~Hz})$ associated with increased cortical excitability ${ }^{54,55}$. Recently, another rTMS protocol was approved by the Federal Drug Administration (FDA) called theta burst stimulation (TBS). The TBS is named after using a low-intensity, $50 \mathrm{~Hz}$ pulse train repeated every $200 \mathrm{~ms}(5 \mathrm{~Hz})^{56}$, which mimics the endogenous theta $(4-7 \mathrm{~Hz})$ waves of the brain, and resembles the experimental models of inducing LTP and LTD of synaptic activity. Different patterns of TBS stimulation (continuous or intermittent) produce opposite effects on the stimulated motor cortex ${ }^{56}$. Intermittent TBS (iTBS) applies 600 pulses in three minutes, and increases motor cortical excitability similar to LTP, while continuous TBS (cTBS) reduces it, similar to LTD $^{56}$.

A rTMS protocol involves the selection of these stimulation parameters, and should take into account the desired physiological effect and existing evidence for rTMS treatment of the patient's condition ${ }^{57}$. A rTMS session usually involves application of 600 to 3000 pulses.

- Stimulation frequency:

Typical patterns for a high frequency rTMS involve a 5 second $10 \mathrm{~Hz}$ pulse train, a 20 to 40 seconds pause between pulse trains, with a total of 1200 to 3000 pulses emitted over a time interval of 15 to 45 minutes. This pause between trains is recommended, and its time must conform to the most current safety guidelines ${ }^{58}$. Low frequency rTMS does not require pause between trains, so a stimulation session consists of a $1 \mathrm{~Hz}$ pulse sequence with 600 to 1500 pulses emitted in a relatively shorter interval.

TBS consists of the application of a $5 \mathrm{~Hz}$ train of 3 to $550 \mathrm{~Hz}$ pulses. For iTBS, of excitatory effect, 2 second interval of $50 \mathrm{~Hz}$ pulses followed by an 8 second pause is usually applied, with a session of 1200 pulses lasting less than 8 minutes. The cTBS, of inhibitory effect, does not use pauses, with a session of 1200 pulses emitted in approximately 3 minutes ${ }^{56}$.

There are also other interventions that combine protocols of high and low frequencies in the same session. One method is called a bilateral rTMS, which applies high frequency stimulation on one side of the brain simultaneously, or more often sequentially, to the low frequency stimulation on the other side. Another method is called priming, when a specific protocol is applied in one particular brain region before another, in order to intensify the effects of the second protocol ${ }^{59}$.

- Resting motor threshold (RMT):

The resting motor threshold is classically defined as the smallest percentage of the maximum field strength required to generate a MEP of $50 \mu \mathrm{V}$ or more in at least 5 of 10 attempts. The RMT is usually determined by stimulating the region of the motor cortex that controls hand movements and performing EMG on one of the small muscles of the hand. It is important to determine the RMT in order to have a field strength intensity that causes depolarization of the motor cortex, thus extrapolating this stimulation effect to other cortical regions. Usually, rTMS protocols use intensities of 100 to $120 \%$ of the RMT, while TBS protocols use intensities of 80 to $100 \%$.

- Field coil:

The shape of the coil is related to the shape of the generated magnetic field, which in turn determines the intensity, precision and depth of the stimulation. The most commonly used field coil is the 8-shaped coil, which creates a heavily focused magnetic field in the center. Also used are H-shaped and double-cone coils.

\section{- Coil positioning:}

The cortical target of stimulation is a crucial parameter for the therapeutic effect of TMS, since the desired physiological effects depend on properly stimulating the stipulated areas. When determining the region to be stimulated, it is important to position the coil correctly so that the generated field reaches that region. The gold standard for targeting is through neuronavigation, which consists of using magnetic resonance imaging of the patient's brain integrated into a spatial positioning system and a group of sensors by software that indicates the relative position of the sensors to the brain. In the absence of neuronavigation, anatomical methods can be used. These methods use cranial reference points to estimate the target. The most widely used of these is the international 10-20 system for electroencephalography (EEG).

\section{Transcranial Current Stimulation (tDCS)}

The main parameters of tDCS are the intensity of the electrical current administered, the duration of the stimulation session, the size and positioning of the electrodes on the scalp, the number of sessions and the 
interval between sessions ${ }^{60}$. The currents applied are between 0.5 and $2 \mathrm{~mA}$; sessions last from 9 to 30 minutes; the size of the electrodes ranges from 3.5 to $100 \mathrm{~cm}^{2}$, the most common being 25 and $35 \mathrm{~cm}^{2}$. The positioning of the electrodes has to be done in order to direct the current to the target region, with the international 10-20 EEG system being the most common reference for this positioning. Effects resulting from a single stimulation session usually last a few minutes, while multiple sessions prolong these effects for up to weeks ${ }^{61}$, and are used clinically. In the scope of the research, different configurations of these parameters are being explored in order to expand our understanding of the technique and also to find new configurations that can be effective as a form of treatment.

\section{Electroconvulsive therapy and Magnetoconvulsive therapy (ECT and MCT) \\ ECT parameters are the positioning of the electrodes,} the intensity and width of the electrical pulses. The most common assemblies are bitemporal (BT), bifrontal (BF) and unilateral right (ULR). The intensity is measured according to the lowest intensity that produces a seizure, called a seizure threshold (ST). Bilateral treatments (BT and BF) usually use 1.5 to 2 times ST, while ULR applies 5 to 8 times $\mathrm{ST}^{38}$. A meta-analysis ${ }^{62}$ compared the three positions and found similar efficacy among them, but with different effects on cognitive functions. BF and ULR ECT are considered first-line treatments, and BT is recommended as a second-line treatment due to a higher index of shortterm adverse effects on cognition ${ }^{38}$. Brief pulses (BP, 0.5 to $1.5 \mathrm{~ms}$ duration) or ultra short pulses (USP, duration less than $0.5 \mathrm{~ms}$ ) are usually used. USP may be associated with lower cognitive impairment, specifically memory $\operatorname{loss}^{63}$, but may also have a slower pace of improvement and require more sessions than $\mathrm{BP}^{64}$. MCT parameters are still under investigation. Most studies position the coil at the apex (equivalent to $\mathrm{Cz}$ in the 10-20 EEG), apply a stimulation frequency of $100 \mathrm{~Hz}$, pulse width of 0.2 to $0.4 \mathrm{~ms}$, and stimulation of 10 seconds. Treatment is usually done 2 to 3 times a week, for a total of 12 sessions $^{38}$.

\section{CONTRAINDICATIONS, ADVERSE EFFECTS AND SAFETY}

The contraindications for rTMS and tDCS are very similar, and include presence of ferromagnetic parts or electronic devices near the stimulation area that can be heated or damaged by induced electric currents.

RTMS may in some cases cause seizures. However, the number of occurrences reported is very small $(<0.1 \%)$, and none of them reported irreversible damage or death $^{58}$. The risk of seizure is associated with higher RMT, higher stimulation frequencies, longer pulse train duration and shorter interval between trains ${ }^{58}$. The experience gained from clinical trials allows the development of increasingly safe protocols in order to establish the limits for the different stimulation parameters. For patients with a previous history of seizures, low frequency rTMS is recommended, which is not associated with a risk of seizures and may even have protective effects ${ }^{66}$. Other adverse effects of rTMS include headache and discomfort at the stimulation site ${ }^{58}$. Adverse effects of ECTS include tingling, paresthesia, redness and discomfort at the site of stimulation.

There are no absolute contraindications for ECT and MCT. The following conditions are associated with higher risks: brain injury, elevated intracranial pressure, recent myocardial infarction, recent cerebral haemorrhage, presence of aneurysm, pheochromocytoma, and class 4 or 5 of anesthesia risk ${ }^{38}$. The most common adverse effects are short-term cognitive impairments, such as spatial disorientation, retrograde and anterograde amnesia, which may last from weeks to a few months, and symptoms that appear during treatment such as headache, muscle aches, and nausea.

\section{CLINICAL EVIDENCE}

Major Depressive Disorder (MDD)

\section{Transcranial Magnetic Stimulation (TMS)}

According to the Canadian Network for Mood and Anxiety Treatments (CANMAT), rTMS is a first-line treatment for patients who have not had improvement after at least one drug treatment. The recommended stimulation parameters are 110 to $120 \%$ of the RMT for high and low frequency rTMS and 70 to $80 \%$ of the RMT for TBS, 5 times a week, for a total of 20 to 30 sessions or less if clinical response is obtained ${ }^{68}$. The stimulated region is the left dorsolateral prefrontal cortex (DLPFC) in the case of high frequency rTMS, or the right DLPFC in the case of low frequency rTMS, both of which are considered first-line protocols. There is a suggestion that patients who do not respond to treatment with low-frequency rTMS in the right DLPFC can respond to high-frequency therapy in the left DLPFC, and vice versa. Thus, a second-line recommendation is to switch non-responsive patients to the other treatment protocol ${ }^{68}$.

The US Food and Drug Administration (FDA) also approved a treatment protocol for MDD using rTMS that involves stimulating the left DLPFC for 37.5 minutes with $10 \mathrm{~Hz}$ pulses (a total of 3000 pulses per session) to $120 \%$ of the RMT, 5 days per week for a period of 4 to 6 weeks $^{69}$. Although it is a standard protocol for MDD treatment of drug-resistant patients, when compared to electroconvulsive therapy (ECT), it has been shown inferior, especially in psychotic patients ${ }^{70}$. In addition, rTMS was shown to be ineffective in patients resistant to ECT, indicating that treatment should not be used if ECT was not successful.

Recently, the FDA also approved a TBS protocol 
Suen PJC, Brunoni AR. Non-invasive brain stimulation therapies.

for the treatment of MDD following a clinical trial that indicated non-inferiority of TBS over the standard highfrequency $r T M S$ protocol ${ }^{71}$. The clinical trial used iTBS applied to left DLPFC with intensity of $120 \%$ of the RMT, $5 \mathrm{~Hz}$ pulse train consisting of $350 \mathrm{~Hz}$ pulses, 2 seconds active and 8 seconds inactive, with a total of 600 pulses emitted in 3 minutes and 9 seconds, 5 days a week for four weeks. The main advantage of this new 3-minute protocol over the 37.5-minute pattern is the shorter stimulation time. Although the number of pulses is very different between the two treatments, the response to treatment by iTBS is similar to that of the standard protocol. In addition, a positive result of the study is that the patients evaluated had drug resistant MDD and did not respond, on average, to one or two previous pharmacological treatments ${ }^{71}$.

\section{Transcranial Current Stimulation (tDCS)}

CANMAT considers tDCS a third line treatment for MDD. This is due to heterogeneous methodologies and inconsistent meta-analysis results. Future studies are needed to establish optimal stimulation parameters for tDCS as monotherapy or in combined therapies ${ }^{68}$.

The role of tDCS as a combined therapy or as a substitute for antidepressants has been investigated in two large clinical trials. Sertraline vs. Electric Current Therapy for Treating Depression Clinical Study (SELECTTDCS $)^{72}$ recruited 120 patients who had not previously used antidepressants, with moderate to severe depression, and randomized them into four groups: sham tDCS and placebo pill, sham tDCS and sertraline, active tDCS and placebo pill, and active tDCS and sertraline. The stimulation parameters were: $2 \mathrm{~mA}, 30$ minutes per day for 2 weeks, followed by two sessions of tDCS every two weeks until the sixth week. Sertraline dose was $50 \mathrm{mg} /$ day. The main conclusions were: 1) combined therapy was significantly more effective than monotherapy; 2) active tDCS as monotherapy was more effective than placebo; 3) tDCS was well tolerated, with few adverse effects, although five cases of mania / hypomania were reported in the combined group.

The findings that tDCS and sertraline are not statistically different are limited because the dose of sertraline was low and the study was not designed to show non-inferiority. The Escitalopram vs. Electric Current Therapy to Treat Depression Clinical Study (ELECTTDCS $)^{73}$ was designed to demonstrate non-inferiority of tDCS to the maximum dosage of escitalopram $(20 \mathrm{mg} /$ day). ELECT-TDCS lasted for 10 weeks (instead of 6 weeks of SELECT-TDCS), and more tDCS sessions were applied (22 instead of 12). ELECT-TDCS failed to demonstrate non-inferiority of tDCS compared to escitalopram, which means that the stimulation was shown as not inferior to the administered antidepressant. Superiority analysis showed that escitalopram was superior to tDCS and placebo, whereas tDCS was superior to placebo. The stimulation protocol used in this study was 5 weekly stimulation sessions in the first 3 weeks, followed by intermittent sessions in the other 7 weeks; a more intense stimulation protocol could have been more effective, in the same way that early studies of rTMS involving two weeks of stimulation showed modest results compared to studies that lasted 4 to 6 weeks.

A multi-center study ${ }^{74}$ involving 130 participants diagnosed with MDD and randomized to receive active tDCS (2.5 mA for 30 minutes) or sham $(0.034 \mathrm{~mA}$ and two 60-second current ramps to mimic the sensation of skin tingling) applied to left DLPFC, administered in 20 sessions in a period of 4 weeks found no significant difference between active and placebo stimulation. In addition, for patients with unipolar depression, placebo resulted in a higher remission rate than active tDCS. This finding points to the possibility that the current applied by the sham stimulation may be biologically active, especially when applied during 20 sessions over 4 weeks. Another important consideration is regarding the ideal "dose" of current. The optimal current intensity for the therapeutic effects of tDCS is not known, and it may be that $2.5 \mathrm{~mA}$ for 30 minutes in 20 sessions exceeds this optimal "dose" for many participants.

\section{Electroconvulsive therapy (ECT)}

ECT is one of the most effective treatments for MDD. Treatment response rates reach $70 \%$, with remission rates of $50 \%$ or higher, depending on the population and the type of stimulus used ${ }^{38}$. For example, a multicenter (n $=230$ ) study reported remission rates of $55 \%$ for ULR, $61 \%$ for BF and $64 \%$ for BT in a mixed group of patients with unipolar (77\%) and bipolar (23\%) depression ${ }^{75}$. The greatest predictor of non-response to ECT is resistance to previous treatments; in patients with degrees of resistance to pharmacological and psychological treatments, the rate of ECT response was approximately 50\%, compared to $65 \%$ in nonresistant patients ${ }^{76}$. The relapse rate, with or without maintenance of treatment, is also high. A meta-analysis of 32 studies from 1962 to 2013 that analyzed the relapse rate after successful treatment with ECT reported that this rate is higher in the 6 months posttreatment $(37.7 \%)^{77}$. Even in those receiving a post-ECT maintenance treatment, relapse rates of approximately $50 \%$ after 1 year were observed.

\section{Schizophrenia}

Noninvasive stimulation techniques have been used to treat auditory hallucinations (AH) and negative symptoms in schizophrenia ${ }^{78-81}$. Patients with $\mathrm{AH}$ have increased blood flow in the left cerebral hemisphere, particularly in the upper temporal gyrus ${ }^{81}$. Thus, solutions that apply low frequency rTMS or cathode tDCS on this region have been investigated. A recent meta-analysis ${ }^{79}$ demonstrated a significant clinical effect of low-frequency rTMS on the left temporoparietal region, but no large 
clinical trial confirmed this finding. In a clinical trial of 30 patients who underwent tDCS, anodic stimulation on TP3 and cathodic on F3 decreased AH for up to three months after application $^{78}$.

High-frequency rTMS has been used to treat negative symptoms of schizophrenia. Pulses are applied to the DLPFC, the brain region that exhibits reduced blood flow and metabolism under these conditions ${ }^{82}$, and although initial results are promising ${ }^{80}$, a recent clinical trial recruiting 156 patients with schizophrenia and exacerbated negative symptoms did not show superiority of high frequency rTMS applied to DLPFC in 105 days of post-treatment follow-up ${ }^{83}$. Studies with small groups of patients receiving tDCS suggest efficacy of an anodal stimulation protocol on the DLPFC and association of clinical improvement with increased connectivity between the DLPFC and left temporal gyrus ${ }^{78,84}$.

\section{Obsessive Compulsive Disorder (OCD)}

The U.S. FDA has approved deep transcranial magnetic stimulation (dTMS), a rTMS modality which targets neural networks that are deeper in the brain by using a more focalized coil, for treatment of OCD. The basis for approval was a clinical trial involving 100 patients which showed that dTMS targeting the dorsomedial prefrontal cortex (DMPFC) and the anterior cingulate cortex significantly improved OCD symptoms ${ }^{85}$. Moreover, there is growing evidence of rTMS efficacy for OCD targeting supplementary motor cortex and the DLPFC ${ }^{86,87}$. No controlled clinical trial evaluating the efficacy of tDCS in OCD has been finalized. A study is investigating the efficacy of cathodal stimulation on the motor supplemental area with $2 \mathrm{~mA}^{88}$. Other studies indicate promising results using this assembly ${ }^{89,90}$.

\section{FUTURE PERSPECTIVES}

New therapeutic targets

The DLPFC is the most common target for treatment of depression by noninvasive techniques. Although it is involved in the pathophysiology of the disease, it was initially targeted because it was easily accessible, and also because our knowledge about the other circuits involved in depression was limited ${ }^{91}$.

It is interesting to note that most of the evidence of prefrontal asymmetry and hypoactivity of DLPFC in depression comes from rTMS studies themselves. The most consistent piece of evidence is that DLPFC is involved in processing information other than emotions, and that, which are not among the main symptoms of the disease ${ }^{91}$. Thus, the antidepressant effects of rTMS on DLPFC may be by indirect mechanisms, through changes in the connectivity of neural networks.

A possible target for future studies of rTMS in depression is the DMPFC. It is a center of convergence of several neural networks involved in cognitive control, regulation of affection and self-concerning thoughts ${ }^{92}$. Injuries in this region cause mood disorders in more than $80 \%$ of cases $^{93}$. A meta-analysis of cerebral morphometry showed a reduction of gray matter in the DMPFC and the anterior cingulate cortex in patients with depression, while reporting few changes of the $\mathrm{DLPFC}^{94}$. Other areas involved in the pathophysiology of depression are the frontopolar cortex (FPC), the prefrontal ventromedial cortex (VMPFC), and the ventrolateral prefrontal cortex (VLPFC), which are hyperactive in depression and are associated with a symptomatic improvement when deactivated ${ }^{91,93}$. The drawback is that VMPFC and VLPFC are not easily accessible, whereas FPC is in a region where stimulation may be uncomfortable by causing muscle contraction of the face, and visual perceptions by stimulating the retina ${ }^{91}$.

\section{Biomarkers}

The use of biomarkers for noninvasive therapies may be useful in identifying groups of patients who have a greater response to rTMS when compared to other interventions or placebo. Two recent studies indicate possible predictors of response. The first study ${ }^{95}$ showed that patients who did not respond to rTMS treatment had higher anhedonia and lower connectivity of a classically reward-associated neural circuit, which is the tegmentum, striatum and part of the VMPFC, at the beginning of treatment. This study identified that a particular subtype of depression in patients, defined from syndromic and neuroimaging characteristics, may respond better to rTMS.

The other study ${ }^{96}$ used functional magnetic resonance imaging in a multicenter sample of 1188 depressed patients and identified four neurophysiological subtypes ("biotypes") based on distinct patterns of dysfunctional connectivity of frontostriatal and frontolimbic networks. Patients of "biotype 1" were approximately three times more likely to benefit from rTMS treatment than patients from "biotypes 2 and 4". The authors suggested that there is a neural signature associated with the clinical response of rTMS.

\section{CONCLUSION}

Noninvasive techniques, such as rTMS and tDCS, the most common methods, are increasingly being applied in clinical practice, due to the research progress in these areas in recent decades. The most promising results are of applications for treating MDD and, to a lesser extent, schizophrenia. Advantages include mild side effects and the absence of impaired contraindications. However, the therapeutic effects of these techniques are still modest, possibly due to the limited understanding of their effects on brain function in healthy and ill patients. The continued development of noninvasive techniques is crucial to elucidate their role in the therapeutic arsenal for treating psychiatric disorders. 
Suen PJC, Brunoni AR. Non-invasive brain stimulation therapies.

\section{REFERENCES}

1. Bussel B. History of electrical stimulation in rehabilitation medicine. Ann Phys Rehabil Med. 2015:58;198-200. doi: 10.1016/j.rehab.2015.04.008.

2. Fregni F, Pascual-Leone A. Technology insight: noninvasive brain stimulation in neurology-perspectives on the therapeutic potential of rTMS and tDCS. Nat Clin Pract Neurol. 2007;3(7):383-93. DOI: 10.1038/ncpneuro0530.

3. Galvani L, Aldini G. Commentary on the effect of electricity on muscular motion [De Viribus Electricitatis In Motu Musculari Commentarius] (R.M. Green, Trans.). Vol. E, Licht. Cambridge, Massachusetts; 1791.

4. Piccolino M. Luigi Galvani and animal electricity: two centuries after the foundation of electrophysiology. Trends Neurosci. 1997;20:443-8. https://doi.org/10.1016/S01662236(97)01101-6

5. Fritsch G, Hitzig E. Electric excitability of the cerebrum (T. Crump \& S. Lama, Trans.). Epilepsy Behav. 1870;15:12330.

6. Ferrier D. The localization of function in the brain [Abstract]. Proc R Soc Lond. 1874;22:228-32.

7. Munk H. On the functions of the vortex (Von Bonin, Trans.). In: Thomas CC, Von Bonin G, editor. Some papers on the cerebral cortex. Springfield, Illinois; 1881. p. 97-117.

8. Rabagliati A. Luciani and Tamburini on the functions of the brain: psycho-sensory cortical centres. Brain. 1879;1:52944.

9. Gross CG. Brain, Vision, memory: tales in the history of neuroscience. Cambridge: MIT Press; 1998.

10. Lewis PM, Rosenfeld JV. Electrical stimulation of the brain and the development of cortical visual prostheses: an historical perspective. Brain Res. 2016;1630:208-24. doi: 10.1016/j.brainres.2015.08.038

11. Bindman LJ, Lippold OC, Redfearn JW. The action of brief polarizing currents on the cerebral cortex of the rat (1) during current flow and (2) in the production of long-lasting after-effects. J Physiol. 1964;172:369-82. doi: 10.1113/ jphysiol.1964.sp007425.

12. Purpura DP, McMurtry JG. Intracellular activities and evoked potential changes during polarization of motor cortex. J Neurophysiol. 1965;28(1):166-85. doi: 10.1152/ jn.1965.28.1.166.

13. Barker AT, Jalinous R, Freeston IL. Non-invasive magnetic stimulation of human motor cortex. Lancet. 1985;1:11067. Doi: 10.1016/s0140-6736(85)92413-4.

14. Nitsche MA, Paulus W. Excitability changes induced in the human motor cortex by weak transcranial direct current stimulation. J Physiol. 2000;527:633-9. doi: 10.1111/j.1469-7793.2000.t01-1-00633.x.

15. Thompson SP. Michael Faraday: his life and work. London: Cassel and Company; 1898.

16. George MS, Aston-Jones G. Noninvasive techniques for probing neurocircuitry and treating illness: vagus nerve stimulation (VNS), transcranial magnetic stimulation (TMS) and transcranial direct current stimulation (tDCS). Neuropsychopharmacology. 2010;35:301-16. doi: 10.1038/ npp.2009.87.
17. Hallett M. Transcranial magnetic stimulation: a primer. Neuron. 2007;55:187-99. doi: 10.1016/j.neuron.2007.06.026

18. Cooke SF, Bliss TV. Plasticity in the human central nervous system. Brain. 2006;129:1659-73. doi: 10.1093/brain/ aw1082.

19. Karabanov A, Ziemann U, Hamada M, George MS, Quartarone A, Classen J, et al. Consensus paper: probing homeostatic plasticity of human cortex with non-invasive transcranial brain stimulation. Brain Stimul. 2015;8:442-54. doi: 10.1016/j.brs.2015.01.404.

20. Priori A,Berardelli A, Rona S, Accornero N, Manfredi M. Polarization of the human motor cortex through the scalp. Neuroreport. 1998;9:2257-60. doi: 10.1097/00001756199807130-00020.

21. Nitsche MA, Paulus W. Sustained excitability elevations induced by transcranial DC motor cortex stimulation in humans. Neurology. 2001;57:1899-901. doi: 10.1212/ wnl.57.10.1899.

22. Liebetanz D, Nitsche MA, Tergau F, Paulus W. Pharmacological approach to the mechanisms of transcranial DC-stimulation-induced after-effects of human motor cortex excitability. Brain. 2002;125:2238-47. doi: 10.1093/ brain/awf238.

23. Nitsche MA, Fricke K, Henschke U, Schlitterlau A, Liebetanz D, Lang N, et al. Pharmacological modulation of cortical excitability shifts induced by transcranial direct current stimulation in humans. J Physiol. 2003; 553:293301. doi: 10.1113/jphysiol.2003.049916.

24. Stagg CJ, Best GJ, Stephenson MC, O'Shea J, Wylezinska M, Kincses ZT, et al. Polarity-sensitive modulation of cortical neurotransmitters by transcranial stimulation. J Neurosci. 2009;29:5202-06. doi: 10.1523/ JNEUROSCI.4432-08.2009.

25. Polanía R, Nitsche MA, Paulus W. Modulating functional connectivity patterns and topological functional organization of the human brain with transcranial direct current stimulation. Hum Brain Mapp. 2011;32(8):1236-49. doi: 10.1002/hbm.21104.

26. Keeser D, Meindl T, Bor J, Palm U, Pogarell O, Mulert $\mathrm{C}$, et al. Prefrontal transcranial direct current stimulation changes connectivity of resting-state networks during fMRI. J Neurosci. 2011;31:15284-93. doi: 10.1523/ JNEUROSCI.0542-11.2011.

27. Jefferys JG. Nonsynaptic modulation of neuronal activity in the brain: electric currents and extracellular ions. Physiol Rev. 1995;75:689-723. doi: 10.1152/physrev.1995.75.4.689.

28. Ardolino G, Bossi B, Barbieri S, Priori A. Nonsynaptic mechanisms underlie the after-effects of cathodal transcutaneous direct current stimulation of the brain. J Physiol. 2005; 568:653-63. doi: 10.1113/ jphysiol.2005.088310

29. Wiethoff S, Hamada M, Rothwell JC. Variability in response to transcranial direct current stimulation of the motor cortex. Brain Stimul. 2014;7(3):468-75. doi: 10.1016/j. brs.2014.02.003.

30. Shekhawat GS, Sundram F, Bikson M, Truong D, De Ridder $\mathrm{D}$, Stinear CM, et al. Intensity, duration, and location of high-definition transcranial direct current stimulation for tinnitus relief. Neurorehabil Neural Repair. 2016; 30:34959. doi: $10.1177 / 1545968315595286$. 
31. Batsikadze G, Moliadze V, Paulus W, Kuo MF, Nitsche MA. Partially non-linear stimulation intensity-dependent effects of direct current stimulation on motor cortex excitability in humans. J Physiol. 2013;591(7):1987-2000. doi: 10.1113/ jphysiol.2012.249730.

32. Monte-Silva K, Kuo MF, Hessenthaler S, Fresnoza S, Liebetanz D, Paulus W, Nitsche MA. Induction of late LTPlike plasticity in the human motor cortex by repeated noninvasive brain stimulation. Brain Stimul. 2013; 6(3):424-32. doi: 10.1016/j.brs.2012.04.011.

33. Lefaucheur JP. Neurophysiology of cortical stimulation. Int Rev Neurobiol. 2012;107:57-85. doi: 10.1016/B978-0-12404706-8.00005-X.

34. Faria P, Hallett M, Miranda PC. A finite element analysis of the effect of electrode area and inter-electrode distance on the spatial distribution of the current density in tDCS. J Neural Eng. 2011;8(6):066017. doi: 10.1088/17412560/8/6/066017.

35. Saturnino GB, Antunes A, Thielscher A. On the importance of electrode parameters for shaping electric field patterns generated by tDCS. Neuroimage. 2015;120:25-35. doi: 10.1016/j.neuroimage.2015.06.067.

36. Laakso I, Tanaka S, Mikkonen M, Koyama S, Sadato N, Hirata A. Electric fields of motor and frontal tDCS in a standard brain space: a computer simulation study. NeuroImage. 2016;137:140-51. doi: 10.1016/j. neuroimage.2016.05.032.

37. Brunoni AR, Shiozawa P, Truong D, Javitt DC, Elkis H, Fregni F, et al. Understanding tDCS effects in schizophrenia: a systematic review of clinical data and an integrated computation modeling analysis. Expert Rev Med Devices. 2014; 11:383-94. doi: 10.1586/17434440.2014.911082.

38. Milev RV, Giacobbe P, Kennedy SH, Blumberger DM, Daskalakis ZJ, Downar J, Modirrousta M, et al. Canadian Network for Mood and Anxiety Treatments (CANMAT) 2016 Clinical Guidelines for the Management of Adults with Major Depressive Disorder: section 4. Neurostimulation treatments. Can J Psychiatry. 2016;61(9):561-75. doi: $10.1177 / 0706743716660033$

39. Cretaz E, Brunoni AR, Lafer B. Magnetic Seizure Therapy for Unipolar and Bipolar Depression: A Systematic Review. Neural Plast. 2015;2015:521398. doi: 10.1155/2015/521398.

40. McClintock SM, Tirmizi O, Chansard M, Husain MM. A systematic review of the neurocognitive effects of magnetic seizure therapy. Int Rev Psychiatry. 2011;23(5):413-23. doi: 10.3109/09540261.2011.623687

41. van Buel EM, et al. Immune and neurotrophin stimulation by electroconvulsive therapy: is some inflammation needed after all? Transl. Psychiatry. 2015;5:e609. doi: 10.1038/ tp.2015.100.

42. Hestad KA, Tønseth S, Støen CD, Ueland T, Aukrust P. Raised plasma levels of tumor necrosis factor $\alpha$ in patients with depression: normalization during electroconvulsive therapy. J ECT. 2003;19(4):183-8. Available from: https:// insights.ovid.com/pubmed?pmid=14657769.

43. Järventausta $\mathrm{K}$, et al. Changes in interleukin-6 levels during electroconvulsive therapy may reflect the therapeutic response in major depression. Acta Psychiatr Scand. 2017;135(1):87-92. doi: 10.1111/acps. 12665.

44. Roman A, Kreiner G, Nalepa I. Macrophages and depression--a misalliance or well-arranged marriage? Pharmacol Rep. 65, 1663-1672 (2013).

45. Yrondi A, Sporer M, Péran P, Schmitt L, Arbus C, Sauvaget A. Electroconvulsive therapy, depression, the immune system and inflammation: A systematic review. Brain Stimul. 2018;11(1):29-51. doi: 10.1016/j.brs.2017.10.013.

46. Brunoni AR, Baeken C, Machado-Vieira R, Gattaz WF, Vanderhasselt MA. BDNF blood levels after electroconvulsive therapy in patients with mood disorders: a systematic review and meta-analysis. World J Biol Psychiatry. 20141;15(5):411-8. doi: 10.3109/15622975.2014.892633

47. Wilkinson ST, Sanacora G, Bloch MH. Hippocampal volume changes following electroconvulsive therapy: a systematic review and meta-analysis. Biol Psychiatry Cogn Neurosci Neuroimaging. 2017;2(4):327-35. doi: 10.1016/j. bpsc.2017.01.011.

48. Takamiya A, Chung JK, Liang KC, Graff-Guerrero A, Mimura M, Kishimoto T. Effect of electroconvulsive therapy on hippocampal and amygdala volumes: systematic review and meta-analysis. Br J Psychiatry. 2018;212(1):1926. doi: 10.1192/bjp.2017.11

49. Bolwig TG. Neuroimaging and electroconvulsive therapy: a review. J ECT. 2014;30(2):138-42. doi: 10.1097/ YCT.0000000000000140

50. Klomjai W, Katz R, Lackmy-Vallée A. Basic principles of transcranial magnetic stimulation (TMS) and repetitive TMS (rTMS). Ann Phys Rehab Med. 2015;58(4):208-13. doi: 10.1016/j.rehab.2015.05.005.

51. Rossini PM, Rossi S. Transcranial magnetic stimulation: diagnostic, therapeutic, and research potential. Neurology. 2007;68(7):484-8. doi: 10.1212/01.wn1.0000250268.13789. b2.

52. Radhu N, Blumberger DM, Daskalakis ZJ. Cortical inhibition and excitation in neuropsychiatric disorders using transcranial magnetic stimulation. In: Brunoni AR, Nitsche M, Loo C, editors. Transcranial direct current stimulation in neuropsychiatric disorders. Cham: Springer International Publishing; 2016.

53. Hoffman RE, Cavus I. Slow Transcranial magnetic stimulation, long-term depotentiation and brain hyperexcitability disorders. Am J Psychiatry. 2002;159:1093102. doi: 10.1176/appi.ajp.159.7.1093.

54. Berardelli A, Inghilleri M, Rothwell JC, et al. Facilitation of muscle evoked responses after repetitive stimulation in man. Exp Brain Res. 1998;122:79-84. doi: 10.1007/ s002210050493.

55. Pascual-Leone A, Tormos JM, Keenan J, Tarazona F, Canete C, Catala MD. Study and modulation of human cortical excitability with transcranial magnetic stimulation. J Clin Neurophysiol. 1998;15:333-43. Available from: https:// journals.lww.com/clinicalneurophys/Fulltext/1998/07000/ Study_and_Modulation_of_Human_Cortical.5.aspx.

56. Huang YZ, Edwards MJ, Rounis E, Bhatia KP, Rothwell JC. Theta burst stimulation of the human motor cortex. Neuron. 2005;45:201-6. doi: 10.1016/j.neuron.2004.12.033.

57. Lefaucher JP, Andre-Obadia N, Antal A, Ayache SS, Baeken C, Benninger DH, et al. Evidence-based guidelines on the therapeutic use of repetitive transcranial magnetic stimulation (rTMS). Clin Neuro-Physiol. 2014;125:2150206. doi: 10.1016/j.clinph.2014.05.021. 
Suen PJC, Brunoni AR. Non-invasive brain stimulation therapies.

58. Rossi S, Hallett M, Rossini PM, Pascual-Leone A, Safety of TMS Consensus Group. Safety, ethical considerations, and application guidelines for the use of transcranial magnetic stimulation in clinical practice and research. Clin Neurophysiol. 2009;120:2008-39. doi: 10.1016/j. clinph.2009.08.016.

59. Iyver MB, Schleper N, Wassermann EM. Priming stimulation enhances the depressant effect of lowfrequency repetitive transcranial magnetic stimulation. J Neurosci. 2003;23:10867-72. doi: https://doi.org/10.1523/ JNEUROSCI.23-34-10867.2003

60. Dedoncker J, Brunoni AR, Baeken C, Vanderhasselt MA. A systematic review and meta-analysis of the effects of transcranial direct current stimulation (tDCS) over the dorsolateral prefrontal cortex in healthy and neuropsychiatric samples: influence of stimulation parameters. Brain Stimul. 2016;9:501-17. doi: 10.1016/j. brs.2016.04.006.

61. Boggio PS, Rigonatti SP, Ribeiro RB, Myczkowski ML, Nitsche MA, Pascual-Leone A, et al. A randomized, doubleblind clinical trial on the efficacy of cortical direct current stimulation for the treatment of major depression. Int $\mathrm{J}$ Neuropsychopharmacol. 2008;11:249-54. doi: 10.1017/ S1461145707007833.

62. Dunne RA, McLaughlin DM. Systematic review and meta-analysis of bifrontal electroconvulsive therapy versus bilateral and unilateral electroconvulsive therapy in depression. World J Biol Psychiatry. 2012;13:248-58. doi: 10.3109/15622975.2011.615863.

63. Verwijk E, Comijs HC, Kok RM. Neurocognitive effects after brief pulse and ultrabrief pulse unilateral electroconvulsive therapy for major depression: a review. J Affect Disord. 2012;140:233-43. doi: 10.1016/j. jad.2012.02.024.

64. Loo CK, Garfield JB, Katalinic N. Speed of response in ultrabrief and brief pulse width right unilateral ECT. Int J Neuropsychopharmacol. 2013;16:755-61. doi: 10.1017/ S1461145712000806.

65. Lefaucheur JP, Antal A, Ayache SS, Benninger DH, Brunelin J, Cogiamanian F, Cotelli M, et al. Evidencebased guidelines on the therapeutic use of transcranial direct current stimulation (tDCS). Clin Neurophysiol. 2017;128(1):56-92. doi: 10.1016/j.clinph.2016.10.087.

66. Fertonani A, Rosini S, Cotelli M, Rossini PM, Miniussi C. Naming facilitation induced by transcranial direct current stimulation. Behav Brain Res. 2010;208:311-8. doi: 10.1016/j.bbr.2009.10.030.

67. Brunoni AR, Chaimani A, Moffa AH, Razza LB, Gattaz WF, Daskalakis ZJ, et al. Repetitive transcranial magnetic stimulation for the acute treatment of major depressive episodes: a systematic review with network metaanalysis. JAMA Psychiatry. 2017;74:143-52. doi: 10.1001/ jamapsychiatry.2016.3644.

68. Milev RV, Giacobbe P, Kennedy SH, Blumberger DM, Daskalakis ZJ, Downar J, et al. Canadian network for mood and anxiety treatments (CANMAT) 2016 clinical guidelines for the management of adults with major depressive disorder: Section 4. neurostimulation treatments. Can J Psychiatry. 2016;61:561-75. doi: 10.1177/0706743716660033.

69. O'Reardon JP, Solvason HB, Janicak PG et al. Efficacy and safety of transcranial magnetic stimulation in the acute treatment of major depression: a multisite randomized controlled trial. Biol Psychiatry. 2007;62:1208-16.

70. Ren J, Li H, Palaniyappan L, Liu H, Wang J, Li C, et al. Repetitive transcranial magnetic stimulation versus electroconvulsive therapy for major depression: a systematic review and meta-analysis. Prog Neuropsychopharmacol Biol Psychiatry. 2014;51:181-9. doi: 10.1016/j. pnpbp.2014.02.004.

71. Blumberger DM, Vila-Rodriguez F, Thorpe KE, Feffer K, Noda Y, Giacobbe P, et al. Effectiveness of theta burst versus high-frequency repetitive transcranial magnetic stimulation in patients with depression (THREE-D): a randomized noninferiority trial. Lancet. 2018;391:1683-92. doi: 10.1016/ S0140-6736(18)30295-2.

72. Brunoni AR, Valiengo L, Baccaro A, Zanao TA, de Oliveira JF, Goulart A, et al. The sertraline vs. electrical current therapy for treating depression clinical study: results from a factorial, randomized, controlled trial. JAMA Psychiatry. 2013;70:383-91. doi: 10.1001/2013.jamapsychiatry.32.

73. Brunoni AR, Moffa AH, Sampaio-Junior B, Borrione L, Moreno ML, Fernandes RA, et al. Trial of electrical directcurrent therapy versus escitalopram for depression. N Engl J Med. 2017;376:2523-33. doi: 10.1056/NEJMoa1612999.

74. Loo CK, Husain MM, McDonald WM, Aaronson S, O'Reardon JP, Alonzo A, et al. International randomizedcontrolled trial of transcranial direct current stimulation in depression. Brain Stimul. 2018;11:125-33. doi: 10.1016/j. brs.2017.10.011

75. Kellner CH, Knapp R, Husain MM. Bifrontal, bitemporal and right unilateral electrode placement in ECT: randomised trial. Br J Psychiatry. 2010;196:226-34. doi: 10.1192/bjp. bp.109.066183.

76. Heijnen WT, Birkenhager TK, Wierdsma AI. Antidepressant pharmacotherapy failure and response to subsequent electroconvulsive therapy: a meta-analysis. J Clin Psychopharmacol. 2010;30:616-9. doi: 10.1097/ JCP.0b013e3181ee0f5f.

77. Jelovac A, Kolshus E, McLoughlin DM. Relapse following successful electroconvulsive therapy for major depression: a meta-analysis. Neuropsychopharmacology. 2013;38:246774. doi: 10.1038/npp.2013.149

78. Brunelin J, Mondino M, Gassab L, Haesebaert F, Gaha L, Suaud-Chagny MF, et al. Examining transcranial directcurrent stimulation (tDCS) as a treatment for hallucinations in schizophrenia. Am J Psychiatry. 2012;169:719-24. doi: 10.1176/appi.ajp.2012.11071091.

79. Slotema CW, Blom JD, van Lutterveld R, Hoek HW, Sommer IE. Review of the efficacy of transcranial magnetic stimulation for auditory verbal hallucinations. Biol Psychiatry. 2014;76:101-10. doi: 10.1016/j. biopsych.2013.09.038.

80. Shi C, Yu X, Cheung EF, Shum DH, Chan RC. Revisiting the therapeutic effect of rTMS on negative symptoms in schizophrenia: a meta-analysis. Psychiatry Res. 2014;215:505-13. doi: 10.1016/j.psychres.2013.12.019.

81. Homan P, Kindler J, Hauf M, Walther S, Hubl D, Dierks T. Repeated measurements of cerebral blood flow in the left superior temporal gyrus reveal tonic hyperactivity in patients with auditory verbal hallucinations: a possible trait marker. Front Hum Neurosci. 2013;7:304. doi: 10.3389/ fnhum.2013.00304. 
82. Hill K, Mann L, Laws KR, Stephenson CM, NimmoSmith I, McKenna PJ. Hypofrontality in schizophrenia: a meta-analysis of functional imaging studies. Acta Psychiatr Scand. 2004;110:243-56. doi: 10.1111/j.16000447.2004.00376.x

83. Wobrock T, Guse B, Cordes J, Wolwer W, Winterer G, Gaebel W, et al. Left prefrontal high-frequency repetitive transcranial magnetic stimulation for the treatment of schizophrenia with predominant negative symptoms: a sham-controlled, randomized multicenter trial. Biol Psychiatry. 2015;77:979-88. doi: 10.1016/j. biopsych.2014.10.009.

84. Mondino M, Jardri R, Suaud-Chagny MF, Saoud M, Poulet E, Brunelin J. Effects of fronto-temporal transcranial direct current stimulation on auditory verbal hallucinations and resting-state functional connectivity of the left temporoparietal junction in patients with schizophrenia. Schizophr Bull. 2016;42:318-26. doi: 10.1093/schbul/sbv114.

85. Carmi L, Tendler A, Bystritsky A, Hollander E, Blumberger DM, Daskalakis J, et al. Efficacy and safety of deep transcranial magnetic stimulation for obsessive-compulsive disorder: a Prospective Multicenter Randomized Double-Blind Placebo-Controlled Trial. Am J Psychiatry. 2019:appiajp201918101180. doi: 10.1176/appi. ajp.2019.18101180.

86. Berlim MT, Neufeld NH, Van den Eynde F. Repetitive transcranial magnetic stimulation (rTMS) for obsessivecompulsive disorder (OCD): an exploratory meta-analysis of randomized and sham-controlled trials. J Psychiatr Res. 2013;47:999-1006. doi: 10.1016/j.jpsychires.2013.03.022.

87. Mantovani A, Simpson HB, Fallon BA, Rossi S, Lisanby SH. Randomized sham-controlled trial of repetitive transcranial magnetic stimulation in treatment-resistant obsessivecompulsive disorder. Int J Neuropsychopharmacol. 2010;13:217-27. doi: 10.1017/S1461145709990435.

88. Silva RM, Brunoni AR, Miguel EC, Shavitt RG. Transcranial direct current stimulation for treatmentresistant obsessive-compulsive disorder: report on two cases and proposal for a randomized, sham-controlled trial. Sao Paulo Med J. 2016;134:446-50. doi: 10.1590/15163180.2016 .0155010716
89. Narayanaswamy JC, Jose D, Chhabra H, Agarwal SM, Shrinivasa B, Hegde A, et al. Successful application of add-on transcranial direct current stimulation (tDCS) for treatment of SSRI resistant OCD. Brain Stimul. 2015;8:6557. doi: 10.1016/j.brs.2014.12.003.

90. D'Urso G, Brunoni AR, Mazzaferro MP, Anastasia A, de Bartolomeis A, Mantovani A. Transcranial direct current stimulation for obsessive-compulsive disorder: a randomized, controlled, partial crossover trial. Depress Anxiety. 2016;33:1132-40. doi: 10.1002/da.22578.

91. Downar J, Daskalakis ZJ. New targets for rTMS in depression: a review of convergent evidence. Brain Stimul. 2013;6:231-40. doi: 10.1016/j.brs.2012.08.006.

92. Bakker N, Shahab S, Giacobbe P, Blumberger DM, Daskalakis ZJ, Kennedy SH, et al. rTMS of the dorsomedial prefrontal cortex for major depression: safety, tolerability, effectiveness, and outcome predictors for $10 \mathrm{~Hz}$ versus intermittent theta-burst stimulation. Brain Stimul. 2015;8:208-15. doi: 10.1016/j.brs.2014.11.002.

93. Koenigs M, Grafman J. The functional neuroanatomy of depression: distinct roles for ventromedial and dorsolateral prefrontal cortex. Behav Brain Res. 2009;201:239-43. doi: 10.1016/j.bbr.2009.03.004.

94. Bora E, Fornito A, Pantelis C, Yucel M. Gray matter abnormalities in major depressive disorder: a meta-analysis of voxel based morphometry studies. J Affect Disord. 2012;138:9-18. doi: 10.1016/j.jad.2011.03.049.

95. Downar J, Geraci J, Salomons TV, Dunlop K, Wheeler $\mathrm{S}, \mathrm{Mc}$ Andrews MP, et al. Anhedonia and rewardcircuit connectivity distinguish non-responders from responders to dorsomedial prefrontal repetitive transcranial magnetic stimulation in major depression. Biol Psychiatry. 2014;76:176-85. doi: 10.1016/j.biopsych.2013.10.026.

96. Drysdale AT, Grosenick L, Downar J, Dunlop K, Mansouri $\mathrm{F}$, Meng Y, et al. Resting-state connectivity biomarkers define neurophysiological subtypes of depression. Nat Med. 2017;23:28-38. doi: 10.1038/nm.4246.

Received: July 28, 2019

Accepted: August 10, 2019 